\title{
Impacto do Diabetes Mellitus sobre o prognóstico da Covid-19
}

\author{
Impact of Diabetes Mellitus on the Covid-19 prognosis \\ Impacto de la Diabetes Mellitus en el prognóstico de Covid-19
}

Recebido: 14/04/2021 | Revisado: 25/04/2021 | Aceito: 26/04/2021 | Publicado: 10/05/2021

\author{
Luís Guilherme da Silva Cavalcante \\ ORCID: https://orcid.org/0000-0002-9762-4770 \\ Universidade Estácio de Teresina, Brasil \\ E-mail: guinutri28@gmail.com \\ Andrea Nunes Mendes de Brito \\ ORCID: https://orcid.org/0000-0002-8270-9018 \\ Universidade Estácio de Teresina, Basil \\ E-mail: drea.nunes@hotmail.com
}

\begin{abstract}
Resumo
O objetivo do presente estudo foi investigar o real impacto do Diabetes Mellitus sobre o desfecho clínico de pacientes acometidos pela COVID-19. Trata-se de uma revisão integrativa da literatura realizada em abril de 2021, analisandose publicações indexadas nas bases de dados Science Direct e PubMed, adotando-se estudos retrospectivos de coorte publicados entre os anos de 2020 e 2021, no idioma inglês. Para a eliminação de estudos duplicados, empregou-se o gerenciador de referências bibliográficas Mendeley Desktop 1.19.8. Dos 10 estudos selecionados, 5 deles foram executados na China, enquanto que os 5 restantes ocorreram no Irã, Inglaterra, Bélgica e Estados Unidos, realizados entre os meses de janeiro e julho de 2020. Os resultados demonstraram um forte impacto do Diabetes Mellitus sobre um pior desfecho clínico em internações por COVID-19. Na presente revisão, evidenciou-se o expressivo impacto que a patogênese do DM exerce sobre as diferentes células inerentes ao sistema imunológico, além do aumento do risco da necessidade de admissão em UTI e do uso de suporte respiratório para conter o agravamento da doença. Contudo, estudos de maior precisão, controle populacional e/ou comorbidades se fazem necessários para uma melhor compreensão dos reais prejuízos da associação do DM a COVID-19.
\end{abstract}

Palavras-chave: Diabetes Mellitus; Prognóstico; Covid-19.

\begin{abstract}
The aim of the present study was to investigate the real impact of Diabetes Mellitus on the clinical outcome of patients affected by COVID-19. This is an integrative literature review carried out in April 2021, analyzing publications indexed in the Science Direct and PubMed databases, adopting retrospective cohort studies published between the years 2020 and 2021, in the English language. To eliminate duplicate studies, the Mendeley Desktop 1.19 .8 bibliographic reference manager was used. Of the 10 selected studies, 5 of them were carried out in China, while the remaining 5 took place in Iran, England, Belgium and the United States, carried out between the months of January and July 2020. The results demonstrated a strong impact of Diabetes Mellitus on a worst clinical outcome in hospitalizations due to COVID-19. In the present review, the significant impact that the pathogenesis of DM has on the different cells inherent to the immune system was evidenced, in addition to the increased risk of the need for admission to the ICU and the use of respiratory support to contain the worsening of the disease. However, studies of greater precision, population control and / or comorbidities are necessary for a better understanding of the real losses of the association between DM and COVID-19.
\end{abstract}

Keywords: Diabetes Mellitus; Prognosis; Covid-19.

\section{Resumen}

El objetivo del presente estudio fue investigar el impacto real de la Diabetes Mellitus en el resultado clínico de los pacientes afectados por COVID-19. Se trata de una revisión integradora de la literatura realizada en abril de 2021, analizando publicaciones indexadas en las bases de datos Science Direct y PubMed, adoptando estudios de cohorte retrospectivos publicados entre los años 2020 y 2021, en idioma inglés. Para eliminar estudios duplicados, se utilizó el administrador de referencias bibliográficas Mendeley Desktop 1.19.8. De los 10 estudios seleccionados, 5 de ellos se realizaron en China, mientras que los 5 restantes se llevaron a cabo en Irán, Inglaterra, Bélgica y Estados Unidos, realizados entre los meses de enero y julio de 2020. Los resultados demostraron un fuerte impacto de Diabetes Mellitus sobre peor evolución clínica en hospitalizaciones por COVID-19. En la presente revisión se evidenció el impacto significativo que tiene la patogenia de la DM en las diferentes células inherentes al sistema inmunológico, además del mayor riesgo de necesidad de ingreso en UCI y el uso de soporte respiratorio para contener el empeoramiento. de la enfermedad. Sin embargo, son necesarios estudios de mayor precisión, control poblacional y / o comorbilidades para comprender mejor las pérdidas reales de la asociación entre DM y COVID-19.

Palabras clave: Diabetes Mellitus; Prognóstico; Covid-19. 


\section{Introdução}

A Coronavirus Disease (COVID-19), doença causada pelo vírus Severe Acute Respiratory Syndrome Coronavirus 2 (SARS-Cov-2), surgiu no fim de 2019 apresentando-se como uma nova variação de pneumonia, da qual se alastrou para mais de 213 países em proporções alarmantes, exigindo destes, medidas extremas de isolamento social para conter uma maior dispersão do patógeno (Zanon et al., 2020; Erener, 2020).

Uma particularidade que desperta bastante atenção em relação as infecções pela variação SARS-Cov-2 se deve a sua elevada capacidade de transmissão e uma notável ligação com uma diversidade de doenças crônicas preexistentes no paciente infectado, denominadas comorbidades, das quais têm sido associadas a um pior prognóstico em internações por COVID-19 (Feitoza et al., 2020).

Entre estas, o Diabetes Mellitus (DM), distúrbio endócrino caracterizado por falhas no metabolismo da glicose, vem sendo associado a elevados índices de mortalidade entre pacientes acometidos pela COVID-19, devido a sua maior capacidade de inflamação metabólica e a liberação de uma diversidade de citocinas aos quais os mesmos encontram-se continuamente predispostos (Silva et al., 2020).

Com isso, sabendo que as manifestações clínicas da COVID-19 podem variar desde um estado assintomática até uma infecção grave de cunho respiratório, exigindo uma elevada demanda em gastos públicos com internações, é imprescindível que haja maiores investigações acerca de comorbidades que possam agravar o quadro clínico e o prognóstico de pacientes hospitalizados devido a patogenia do novo coronavírus (Rocha et al., 2021; Fernandes \& Pereira, 2020).

Neste contexto, o presente estudo teve por objetivo investigar o real impacto do DM sobre o desfecho clínico de pacientes acometidos pela COVID-19.

\section{Metodologia}

Trata-se de uma revisão integrativa da literatura da qual objetivou investigar o impacto do DM sobre a prognóstico da COVID-19. Para a elaboração da questão focal, utilizou-se a estratégia PICO (Acrômio para Patient, Intervetion, Comparation e Outcome) sendo a questão delimitada: "Qual a influência do DM sobre o prognóstico de pacientes acometidos pela COVID19?", onde $\mathrm{P}=$ pacientes diabéticos com COVID-19; I= infecção por COVID-19; C= pacientes não diabéticos com COVID-19" e $\mathrm{O}=$ pior desfecho clínico dos pacientes.

A busca dos estudos foi realizada em abril de 2021, analisando-se publicações indexadas nas bases de dados Science Direct e PubMed. Para a seleção dos descritores, foi efetuada uma consulta aos Descritores em Ciências da Saúde (DECs), disponível no portal da BVS. Os artigos foram procurados baseados nos descritores "Diabetes Mellitus" "Prognóstico" e "Covid-19", sendo conectados posteriormente aos operadores lógicos "AND", "OR" e "AND NOT".

Como critérios de elegibilidade, foram adotados estudos retrospectivos de coorte referentes a temática proposta, publicados entre os anos de 2020 e 2021, no idioma inglês. Foram excluídos artigos de revisão, relatos de casos, série de casos e cartas ao editor. Empregou-se o gerenciador de referências bibliográficas Mendeley Desktop 1.19.8 para a eliminação de estudos duplicados.

A seleção dos artigos identificados durante a busca nas bases de dados foi conduzida por meio da técnica sugerida por Lakatos e Marconi (2003), da qual consiste na leitura de títulos seguidos dos resumos e, posteriormente, o artigo por inteiro para então selecioná-los ou não. Por fim, as referências dos artigos selecionados foram rastreadas visando a inclusão de estudos de potencial interesse. O procedimento foi realizado por dois pesquisadores de forma simultânea e independente, levando-se em consideração os critérios de inclusão e exclusão pré-estabelecidos. Aqueles que houveram discordância foram analisandos em reunião com os autores para uma avaliação e consenso. 
A extração dos dados foi realizada por meio de um protocolo elaborado pelos pesquisadores, no qual foram incluídos os seguintes dados: autor/ano de publicação, país de origem, metodologia e conclusão.

Figura 1. Fluxograma da seleção de estudos.

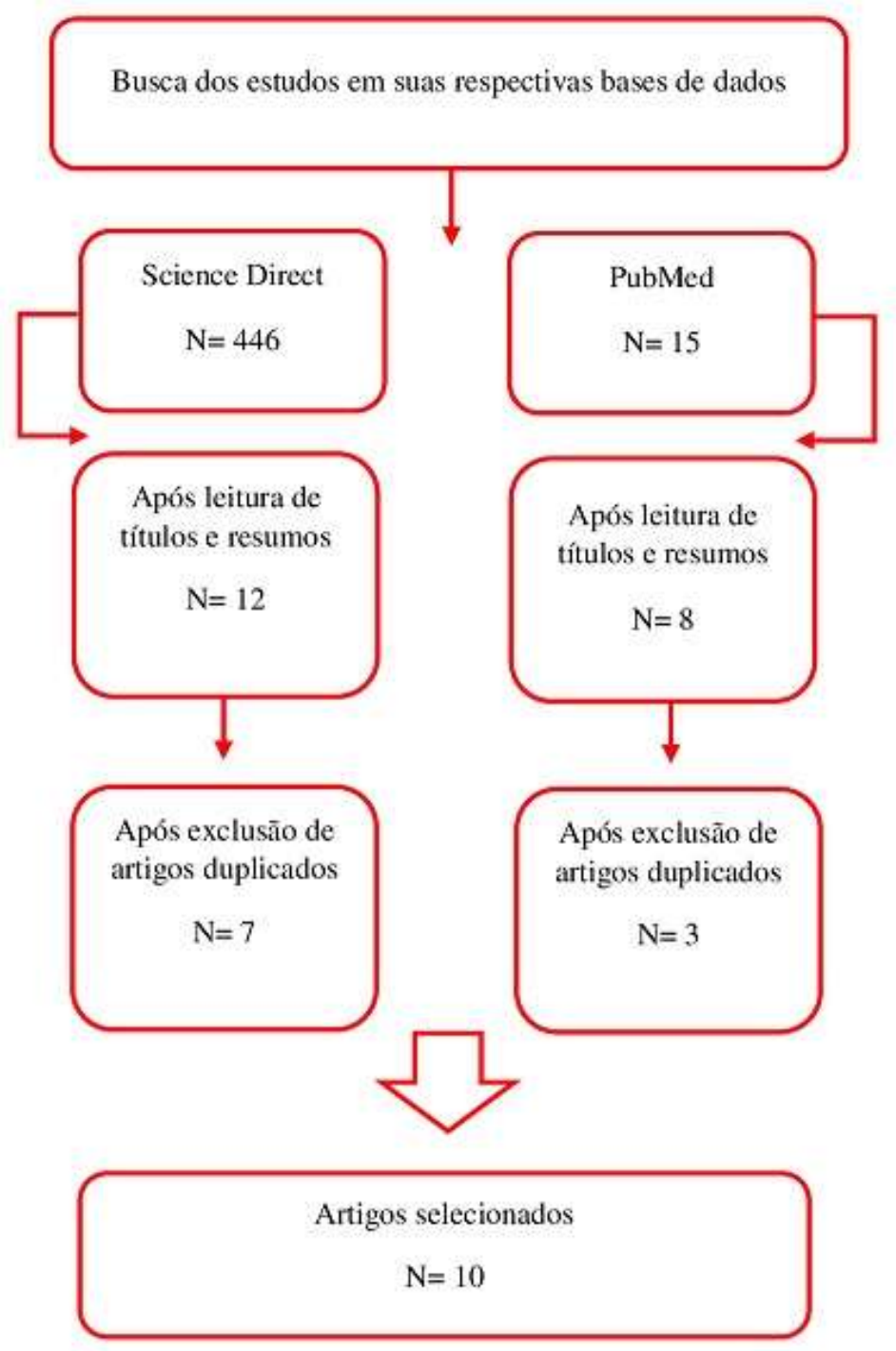

Fonte: Pesquisa direta.

\section{Resultados}

Dos 10 estudos selecionados, 5 deles foram executados na China, enquanto que os 5 restantes ocorreram no Irã, Inglaterra, Bélgica e Estados Unidos, realizados entre os meses de janeiro e julho de 2020. O Quadro 1 apresenta a síntese dos artigos selecionados com os respectivos desfechos referentes ao impacto do DM sobre o prognóstico de internações por COVID-19. 
Quadro 1. características dos estudos selecionados.

\begin{tabular}{|c|c|c|c|}
\hline Autor/ano & País & Metodologia & Conclusão \\
\hline Bode et al., 2020. & Estados Unidos & $\begin{array}{l}\text { Estudo retrospectivo observacional } \\
\text { envolvendo portadores de diabetes. } \\
\text { Para a obtenção dos dados, utilizou-se } \\
\text { o data warehouse de Glytec }\end{array}$ & $\begin{array}{l}\text { Pacientes portadores de diabetes } \\
\text { tiveram um tempo de permanência } \\
\text { hospitalar e índice de mortalidade } \\
\text { maior em relação aos nãos } \\
\text { diabéticos. }\end{array}$ \\
\hline $\begin{array}{l}\text { Akbarigomi; Hosseini; } \\
\text { Rashidiani, } 2020 .\end{array}$ & Irã & $\begin{array}{l}\text { Estudo retrospectivo incluindo } 595 \\
\text { pacientes internados por covid } 19 \mathrm{com} \\
\text { e sem diabetes utilizando-se } \\
\text { parâmetros demográficos, } \\
\text { laboratoriais, clínicos e radiológicos. }\end{array}$ & $\begin{array}{l}\text { Pacientes portadores de diabetes } \\
\text { apresentaram maiores chances de } \\
\text { complicações e mortalidade em } \\
\text { comparação ao grupo sem diabetes. }\end{array}$ \\
\hline Zhang et al., 2020. & China & $\begin{array}{l}\text { Estudo retrospectivo do qual utilizou- } \\
\text { se informações de prontuários e } \\
\text { análises univariadas e multivariadas } \\
\text { para analisar fatores de riscos } \\
\text { associados a eventos graves. }\end{array}$ & $\begin{array}{l}\text { Pacientes portadores de diabetes } \\
\text { apresentaram desfechos clínicos } \\
\text { piores em relação aos não } \\
\text { diabéticos. }\end{array}$ \\
\hline Zhang et al., 2021. & China & $\begin{array}{l}\text { Estudo retrospectivo incluindo } 131 \\
\text { pacientes com e sem diabetes. Foram } \\
\text { utilizados parâmetros demográficos, } \\
\text { histórico médico, sinais e sintomas e } \\
\text { exames laboratoriais. }\end{array}$ & $\begin{array}{l}\text { Os níveis de glicose elevados } \\
\text { associaram-se intimamente ao } \\
\text { agravamento do quadro de } \\
\text { pacientes internados com Covid-19. }\end{array}$ \\
\hline Orioli et al., 2020. & Bélgica & $\begin{array}{l}\text { Estudo retrospectivo de dados da } \\
\text { admissão de pacientes diabéticos } \\
\text { internado com Covid-19. }\end{array}$ & $\begin{array}{l}\text { As taxas de letalidade não } \\
\text { apresentaram resultados } \\
\text { estatisticamente diferentes entre os } \\
\text { pacientes com e sem diabetes. }\end{array}$ \\
\hline Shrestha et al., 2020 & China & $\begin{array}{l}\text { Estudo retrospectivo realizado em } 147 \\
\text { pacientes com e sem diabetes do qual } \\
\text { utilizou-se para a análise dados } \\
\text { demográficos, laboratoriais e } \\
\text { prevalência de sinais e sintomas. }\end{array}$ & $\begin{array}{l}\text { Neste estudo, paciente diabéticos } \\
\text { apresentaram maior taxa de lesão } \\
\text { pulmonar grave e necessidade de } \\
\text { intubação. }\end{array}$ \\
\hline Alkundi et al., 2020. & Inglaterra & $\begin{array}{l}\text { Estudo retrospectivo realizados em } \\
\text { pacientes diabéticos e não diabéticos } \\
\text { utilizando-se como parâmetros o } \\
\text { tempo de permanência no hospital, } \\
\text { status e controle do diabetes. }\end{array}$ & $\begin{array}{l}\text { Pacientes portadores de diabetes } \\
\text { tiveram um tempo de permanência } \\
\text { maior no hospital em relação aos } \\
\text { pacientes não diabéticos. }\end{array}$ \\
\hline Chen et al., 2020. & Estados Unidos & $\begin{array}{l}\text { Estudo retrospectivo incluindo } 208 \\
\text { pacientes com e sem diabetes. Para } \\
\text { obtenção de dados foram utilizados } \\
\text { prontuários médicos incluindo } \\
\text { informações referentes a exames e } \\
\text { evolução dos pacientes. }\end{array}$ & $\begin{array}{c}\text { Pacientes diagnosticados com } \\
\text { diabetes apresentaram maiores } \\
\text { complicações e piores desfechos } \\
\text { clínicos quando comparado aos não } \\
\text { diabéticos. }\end{array}$ \\
\hline Liu; Bai, 2020 & China & $\begin{array}{l}\text { Estudo retrospectivo incluindo } 192 \\
\text { pacientes com e sem diabetes com } \\
\text { dados extraídos de prontuários } \\
\text { médicos até o desfecho clínico destes. }\end{array}$ & $\begin{array}{l}\text { Pacientes diabéticos apresentaram } \\
\text { um maior risco de um pior desfecho } \\
\text { clínico em relação ao grupo } \\
\text { controle. }\end{array}$ \\
\hline Shang et al., 2020. & China & $\begin{array}{l}\text { Para a análise foram extraídos dados } \\
\text { demográficos, clínicos, laboratoriais, } \\
\text { radiológicos, complicações e } \\
\text { desfechos clínicos de pacientes } \\
\text { diabéticos internados por Covid-19. }\end{array}$ & $\begin{array}{l}\text { A presença de diabetes entre os } \\
\text { pacientes apresentou um maior } \\
\text { risco de desfechos clínicos ruins } \\
\text { quando associada a Covid-19. }\end{array}$ \\
\hline
\end{tabular}

Fonte: Pesquisa direta.

\section{Discussão}

Os mecanismos pelos quais o DM influencia negativamente o prognóstico da COVID-19 ainda são obscuros. Aparentemente o distúrbio está associado a ativação do sistema renina-angiotensina e, o consequente aumento da expressão da enzima angiotensina II (ECA2), facilita de forma significativa a entrada do SARS-Cov-2 no organismo através da ligação do 
vírus a esta, o que poderia explicar a associação do DM a um pior desfecho clínico em internações por COVID-19 (Ma \& Holt, 2020).

Neste contexto, estudos retrospectivos realizados nos Estados Unidos envolvendo pacientes diabéticos ou com glicemia mal controlada mostraram que a frequência de anormalidades em varreduras do tórax, identificadas através de tomografia computadorizada (TC) (Chen et al., 2020), o período de permanência hospitalar e o índice de mortalidade entre eles foram substancialmente maiores em relação aos pacientes não portadores de DM (Bode et al., 2020).

Resultados semelhantes foram encontrados no estudo de Akbarigomi et al. (2020), ao identificarem uma maior proporção de anormalidades no tórax achados na TC. Além disso, foram identificadas uma maior contagem de leucócitos, neutrófilo e proteína $\mathrm{C}$ reativa entre os diabéticos, incluindo ainda uma necessidade aumentada do uso de suporte respiratório devido a complicações associadas a COVID-19. Corroborando a estes achados, o estudo de Oriolli et al. (2020) ratificou que a contagem elevada de leucócitos foi um dos principais fatores preditivos para morte relacionada a COVID-19 em pacientes com DM. No entanto, neste último, a taxa de letalidade não apresentou diferenças estatísticas relevantes em relação ao grupo não diabético.

Os riscos de desenvolver a forma letal da COVID-19 ou de uma maior necessidade de entrada em Unidades de Terapia Intensiva (UTI) se mostraram extremamente elevados em uma população diabética na Escócia quando comparada aos indivíduos sem a comorbidade (Gurnaghan et al., 2020) e, além de experimentarem manifestações graves da patogenia do SARS-Cov-2, um estudo retrospectivo realizado em Bangladesh relatou uma série de complicações pós COVID-19 em pacientes com DM, incluindo dor, desconforto e distúrbios relacionados ao sono, pressupondo-se que as reações envolvendo coexistência da patologia podem não se limitarem ao período de internação. (Akter et al., 2020).

Contudo, os desfechos clínicos insatisfatórios podem, ainda, estar relacionados a possíveis interferências do DM sobre o sistema imunológico. Zhang et al. (2020) investigaram 52 pacientes diabéticos internados por COVID-19. Entre estes, 40,4\% desenvolveram a forma severa da doença, apresentando complicações associadas a um elevado risco de vida, seguido de contagens maiores de leucócitos e neutrófilos, e uma menor contagem de linfócitos, além de níveis aumentados de proteína C reativa, a qual foi diretamente ligada a incidência de eventos ruins durante o período de internação. A coexistência de DM também se mostrou como fator chave para o desenvolvimento de pneumonia grave, funcionamento anormal de órgãos e um maior risco de progressão da COVID-19 (Zhang et al., 2021).

Ademais, estudos retrospectivos executados na China reiteraram uma maior necessidade de admissão de pacientes com DM na UTI, maiores taxa de intubação e necessidade do uso de bloqueadores neuromusculares (Shrestha et al., 2020), baixa contagem de linfócitos, hipoalbuminemia, acompanhada de uma maior incidência de pneumonia bilateral e complicações envolvendo insuficiência respiratória e lesão cardíaca, além de um tempo de sobrevida significativamente menor em relação ao grupo não diabético com COVID-19 (Shang et al., 2020).

Após a análise dos estudos, é notório o impacto que o DM exerce sobre os desfechos clínicos de pacientes internados por COVID-19. Todavia, a coexistência de outras comorbidades alheias ao DM entre os indivíduos inclusos em algumas pesquisas, como a hipertensão arterial, pode ter interferido em seus resultados, tornando-se um fator limitante para os achados da presente revisão. Além do mais, em alguns estudos, o número de pacientes diabéticos era relativamente maior em relação aos grupos sem DM, o que poderia implicar em uma incerteza sobre a real influência da síndrome sobre o prognóstico da patogênese do SARS-Cov-2, frisando uma necessidade de estudos com maior controle populacional.

\section{Conclusão}

A coexistência de DM como comorbidade associada a COVID-19 expressou uma forte ligação com piores desfechos clínicos em internações por SARS-Cov-2, configurando-se como um elemento chave a ser investigado em pesquisas futuras. 
Na presente revisão, evidenciou-se o expressivo impacto que a patogênese do DM exerce sobre as diferentes células inerentes ao sistema imunológico, além do aumento do risco da necessidade de admissão em UTI e do uso de suporte respiratório para conter o agravamento da doença. Contudo, estudos de maior precisão, controle populacional e/ou comorbidades se fazem necessários para uma melhor compreensão dos reais prejuízos da associação do DM a COVID-19.

Em conclusão, espera-se que os resultados do presente estudo realce a necessidade de maiores investigações acerca do impacto de comorbidades, em especial o DM sobre o prognóstico de infecções por SARS-Cov-2, uma vez que esta associação se relacionou a um maior tempo de permanência hospitalar e, consequentemente, maiores investimentos financeiros em internações.

\section{Referências}

Rocha, G. V., Soares, C. E. M., de Oliveira Filho, L. H., do Amaral, M. V. F., Castro, V. E., Junior, E. A., \& Amâncio, N. D. F. G. (2021). A influência da obesidade na mortalidade de adultos com COVID-19. Brazilian Journal of Health Review, 4(1), 1405-1418. https://doi.org/10.34119/bjhrv4n1-119.

de Araújo Silva, Á. L. D., Matias, L. D. M., da Silva Freitas, J. M., de Oliveira, J. C. N., \& de Andrade, L. L. (2020). Medidas de prevenção da covid-19 em pessoas que vivem com diabetes mellitus. Revista Enfermagem Atual In Derme, 93, e020004-e020004. https://doi.org/10.31011/reaid-2020-v.93-n.0-art.808.

Feitoza, T. M. O., Chaves, A. M., Muniz, G. T. S., da Cruz, M. C. C., \& Junior, I. D. F. C. (2020). COMORBIDADES E COVID-19. Revista Interfaces: Saúde, Humanas e Tecnologia, 8(3), 711-723. http://dx.doi.org/10.16891/2317-434X.v8.e3.a2020.pp711-723.

Fernandes, G. A. D. A. L. \& Pereira, B. L. S. (2020). Os desafios do financiamento do enfrentamento à COVID-19 no SUS dentro do pacto federativo. Revista de Administração Pública, 54(4), 595-613. https://doi.org/10.1590/0034-761220200290.

Erener, S. (2020). Diabetes, infection risk and COVID-19. Molecular Metabolism, 101044. https://doi.org/10.1016/j.molmet.2020.101044.

Marconi, M. D. A., \& Lakatos, E. M. (2003). Fundamentos de metodologia científica. Atlas.

Zanon, C., Dellazzana-Zanon, L. L., Wechsler, S. M., Fabretti, R. R., \& Rocha, K. N. D. (2020). COVID-19: implicações e aplicações da Psicologia Positiva em tempos de pandemia. Estudos de Psicologia (Campinas), 37. https://doi.org/10.1590/1982-0275202037e200072.

Bode, B., Garrett, V., Messler, J., McFarland, R., Crowe, J., Booth, R., \& Klonoff, D. C. (2020). Glycemic characteristics and clinical outcomes of COVID-19 patients hospitalized in the United States. Journal of diabetes science and technology, 14(4), 813-821. https://doi.org/10.1177/1932296820924469.

Ma, R. C. W., \& Holt, R. I. G. (2020). COVID - 19 e diabetes. Diabetic Medicine, 37(5), 723-725. https://doi.org/10.1111/dme.14300.

McGurnaghan, S. J., Weir, A., Bishop, J., Kennedy, S., Blackbourn, L. A., McAllister, D. A., \& Health Protection Study Group. (2021). Risks of and risk factors for COVID-19 disease in people with diabetes: a cohort study of the total population of Scotland. The Lancet Diabetes \& Endocrinology, 9(2), 82-93. https://doi.org/10.1016/S2213-8587(20)30405-8.

Akbariqomi, M., Hosseini, M. S., Rashidiani, J., Sedighian, H., Biganeh, H., Heidari, R., \& Kooshki, H. (2020). Clinical characteristics and outcome of hospitalized COVID-19 patients with diabetes: A single-center, retrospective study in Iran. diabetes research and clinical practice, 169 , 108467. https://doi.org/10.1016/j.diabres.2020.108467.

Zhang, N., Wang, C., Zhu, F., Mao, H., Bai, P., Chen, L. L., \& Zhou, M. (2020). Risk factors for poor outcomes of diabetes patients with COVID-19: a singlecenter, retrospective study in early outbreak in China. Frontiers in endocrinology, 11. https://doi.org/10.3389 / fendo.2020.571037.

Akter, F., Mannan, A., Mehedi, H. H., Rob, M. A., Ahmed, S., Salauddin, A., \& Hasan, M. M. (2020). Clinical characteristics and short term outcomes after recovery from COVID-19 in patients with and without diabetes in Bangladesh. Diabetes \& Metabolic Syndrome: Clinical Research \& Reviews, 14(6), 20312038. https://doi.org/10.1016/j.dsx.2020.10.016.

Zhang, P., Wang, M., Wang, Y., Wang, Y., Li, T., Zeng, J., \& Gong, Y. (2021). Risk factors associated with the progression of COVID-19 in elderly diabetes patients. diabetes research and clinical practice, 171, 108550. https://doi.org/10.1016/j.diabres.2020.108550.

Orioli, L., Servais, T., Belkhir, L., Laterre, P. F., Thissen, J. P., Vandeleene, B., \& Hermans, M. P. (2021). Clinical characteristics and short-term prognosis of in-patients with diabetes and COVID-19: A retrospective study from an academic center in Belgium. Diabetes \& Metabolic Syndrome: Clinical Research \& Reviews, 15(1), 149-157. https://doi.org/10.1016/j.dsx.2020.12.020.

Shrestha, E., Charkviani, M., Musurakis, C., Kansakar, A. R., Devkota, A., Banjade, R., \& Nava, G. R. (2021). Type 2 diabetes is associated with increased risk of critical respiratory illness in patients COVID-19 in a community hospital. Obesity medicine, 22, 100316. https://doi.org/10.1016/j.obmed.2020.100316.

Alkundi, A., Mahmoud, I., Musa, A., Naveed, S., \& Alshawwaf, M. (2020). Clinical characteristics and outcomes of COVID-19 hospitalized patients with diabetes in the United Kingdom: a retrospective single centre study. diabetes research and clinical practice, 165 , 108263. https://doi.org/10.1016/j.diabres.2020.108263.

Chen, Y., Chen, J., Gong, X., Rong, X., Ye, D., Jin, Y., \& Guo, J. (2020). Clinical characteristics and outcomes of type 2 diabetes patients infected with COVID-19: A retrospective study. Engineering, 6(10), 1170-1177. https://doi.org/10.1016/j.eng.2020.05.017. 
Research, Society and Development, v. 10, n. 5, e38810515069, 2021

(CC BY 4.0) | ISSN 2525-3409 | DOI: http://dx.doi.org/10.33448/rsd-v10i5.15069

Liu, Z., Bai, X., Han, X., Jiang, W., Qiu, L., Chen, S., \& Yu, X. (2020). The association of diabetes and the prognosis of COVID-19 patients: a retrospective study. Diabetes research and clinical practice, 169, 108386. https://doi.org/10.1016/j.diabres.2020.108386.

Shang, J., Wang, Q., Zhang, H., Wang, X., Wan, J., Yan, Y., \& Lin, J. (2021). The relationship between diabetes mellitus and COVID-19 prognosis: a retrospective cohort study in Wuhan, China. The American journal of medicine, 134(1), e6-e14. https://doi.org/10.1016/j.amjmed.2020.05.033. 